\title{
ASO Author Reflections: Evolving Surgical Role in Metastatic Soft Tissue Sarcoma
}

\author{
James Janopaul-Naylor, MD (1), and Pretesh Patel, MD \\ Department of Radiation Oncology, Winship Cancer Institute of Emory University, Atlanta, GA
}

Soft tissue sarcomas (STS) are a heterogenous group of uncommon malignancies known for their resistance to systemic therapies. For metastatic cancer, the role of local therapies such as surgery or radiation was historically reserved for palliation, but identification of a limited metastatic disease state, be it de novo or induced, has altered that paradigm. Thus the question: what is the role for aggressive local therapy for patients with metastatic STS? The National Comprehensive Cancer Network Guidelines note, "there are no data to support the optimal management of patients [with STS] presenting with metastatic disease." 1 As such, we sought to clarify if there are a subset of patients that may benefit from more aggressive local treatments.

We used the National Cancer Database (NCDB) to see if there was a benefit to surgical resection of metastases in a large cohort $(\mathrm{n}=1124)$ of patients with upfront metastatic STS. ${ }^{2}$ Since the NCDB has limited details on metastases, we used definitive intent resection to primary tumor as a surrogate to exclude patients with widely disseminated disease. We found that addition of metastasectomy increased 5-year OS to $30.8 \%$ compared with resection of primary disease alone (5-year OS $18.2 \%, p<0.001$ ).

Further work is needed to verify these hypothesis-generating observations. Prior work has shown that ablative radiation $^{3}$ or metastasectomy ${ }^{4}$ are safe and effective for other metastatic malignancies. Our work indicates that similar approaches may be beneficial for patients with upfront metastatic STS. Further study of aggressive multimodal management of limited metastatic sarcoma is warranted.

FUNDING There is no specific funding.

DISCLOSURE There are no conflicts of interest to declare.

\section{REFERENCES}

1. National Comprehensive Cancer Network. Soft Tissue Sarcoma (Version 2.2021). https://www.nccn.org/professionals/physician_g 1s/pdf/sarcoma.pdf. Accessed 5 July 2021.

2. Abugideiri M, Janopaul-Naylor J, Switchenko J, et al. Impact of metastasectomy and aggressive local therapy in newly diagnosed metastatic soft tissue sarcoma: an analysis of the NCDB. Ann Surg Oncol. 2021. https://doi.org/10.1245/s10434-021-10466-4.

3. Palma DA, Olson R, Harrow S, et al. Stereotactic ablative radiotherapy for the comprehensive treatment of oligometastatic cancers: long-term results of the SABR-COMET Phase II randomized trial. J Clin Oncol. 2020;38(25):2830-8. https://doi. org/10.1200/JCO.20.00818.

4. Silberhumer GR, Paty PB, Temple LK, et al. Simultaneous resection for rectal cancer with synchronous liver metastasis is a safe procedure. Am J Surg. 2015;209(6):935-42. https://doi.org/10. 1016/j.amjsurg.2014.09.024.

Publisher's Note Springer Nature remains neutral with regard to jurisdictional claims in published maps and institutional affiliations.

(C) Society of Surgical Oncology 2021

First Received: 19 July 2021

Accepted: 19 July 2021;

Published Online: 28 July 2021

J. Janopaul-Naylor, MD

e-mail: jjanopa@emory.edu 